\title{
Correspondence
}

\section{Virtual Objects in Electronic Catalogs: A Human-Computer Interface Issue}

\author{
Benjamin P.-C. Yen, Senior Member, IEEE, and Kenny Ng
}

\begin{abstract}
Web interface design is an important aspect of electronic commerce (EC). However, apart from design frameworks and guidelines for Web-based EC, not much has been done by researchers or practitioners on how electronic catalogs (e-catalogs) influence the users' desirability and satisfaction as purchasers. In this correspondence, we investigate the form of media that represented the most efficient mode to present products to Web users by summarizing and evaluating various existing forms of e-catalogs and their respective responses from Web users. We conclude that a 3-D virtual object (VO) is the most efficient mode of electronic cataloging for Web interface due to a better sense of presence of users, a more attractive and enjoyable media of delivery of useful information to users, and a higher level of engagement of user's memory. A 3-D VO, as a result, generates the highest users' satisfaction, which leads to increased propensity to purchase. Further, we discuss the practical and theoretical research implications of these findings to e-catalogs.
\end{abstract}

Index Terms-Human-computer interaction, presentation style, virtual reality (VR), Web-based catalog.

\section{INTRODUCTION}

Computer usability, which measures the easiness and effectiveness of a computer that could be used by a specific set of users, given a particular level of support, to carry out a fixed set of tasks in a defined environment, possesses the following five attributes: 1) learnability; 2) efficiency; 3) memorability; 4) error; and 5) satisfaction [1]. Assessment of usability usually includes evaluation of the system by performing a certain task on a menu of a word processor [2], understanding a symbolic-based or pictogram-based metaphoric design [3], engaging in a 3-D virtual environment (VE) [4]-[8], or interacting with a Web interface as a place for media coverage [9]. Although most research on computer usability began with a common objective to validate a particular interface, researchers often redeveloped separate guidelines for evaluating interfaces due to diversity of style and media of different interfaces, especially where the utility of different 3-D systems was involved [5].

With the recent development in virtual reality (VR), it is critical for designers to understand the usability of 3-D representations [7] in enhancing computer usability. VR is a term that had been widely accepted by the public, yet much research effort had to be undertaken before VR could actually be achieved [11]. Meanwhile, Zeltzer's three-vector cube of autonomy, interaction, and presence [12] was an effective conceptual tool for characterizing VR. However, in current technologies, the degree of automation of objects in a VR environment is relatively low. Kalawsky [11] therefore suggested that VE was a more appropriate term. An object in a hollow VE was defined as a "virtual object" (VO) that could be used in both immersive VE and

Manuscript received January 8, 2001; revised June 10, 2002, February 21, 2003, June 1, 2005, and December 2, 2005. This work was sponsored in part by Taran Eastman under Grant TEIL 99/00.EG01. This paper was recommended by Associate Editor I. Gu.

The authors are with the School of Business, The University of Hong Kong, Kowloon, Hong Kong.

Color versions of one or more of the figures in this paper are available online at http://ieeexplore.ieee.org.

Digital Object Identifier 10.1109/TSMCA.2007.897698 desktop VR systems. More recently, many researchers [4], [8], [13] were engaged in the development of methodologies (e.g., VRUSE, VRSART and VRISE) for the assessment of usability of VR systems.

Internet technology was adopted to sell products and services on the Web. The clothing and fashion industry, e.g., Land's End [48], started to publish details and images of their products on the Web. Tyrwhitt [49] promoted and exhibited clothes patterns and styles on the Internet by introducing attractive 3-D VOs. Burke [14] argued that these 3-D VOs could be used to entertain, persuade, and manage product categories, as well as to maximize category profitability and store performance. By using a futuristic-looking product interface, Sharper Image [50] increased its sales vastly [15]. Although 3-D VOs appeared to be useful, there were few criteria for assessing the effectiveness of 3-D interfaces in comparison with 2-D designs [7], especially in relation to effectiveness of the media for selling consumer products online [16]. Miles et al. [17] agreed that: "It is not clear, for example, what advantages if any 3D VR will have in e-commerce." Therefore, there was a need to determine how the interface of an e-catalog stimulated the users.

There were mainly six ways for an e-catalog to present a product: 1) text; 2) 2-D image; 3) photorealistic VR object and panorama view; 4) 3-D VO; 5) video clip; and 6) their combinations [18]. Video clip was excluded in our evaluation due to its lack of adoption on the Web as well as its high demand for bandwidth in operation. Text and 2-D images on the other hand are the basic elements of e-catalogs. photorealistic facilities offered by QuicktimeVR (QTVR) and modelbased renderings of Virtual Reality Markup Language (VRML) provided sophisticated tools for extrinsic design representation [5], and they were used for product display such as the Apple iBook [51] and Sony camcorder [52].

In this correspondence, we first review literature with respect to the usability of VE and VR. We also address the trend of content of ecatalogs that was moving from static 2-D images to 3-D models [19] with a customizable feature [20]. We further illustrate a set of response variables based on an extensive review for interface evaluation in VE and VR. Finally, we describe the experiment setting and the empirical tests of the hypotheses with the objective to test the use of 3-D VOs in a systematic manner by measuring the subjective users' experience based on a set of response variables among various presentation styles.

\section{RESPONSE VARIABLES}

There were many aspects to discuss the advantages of using a 3-D VO over a 2-D image. In this section, we provide the definition of theoretical support for the utility of response variables, including the sense of presence, level of attraction, degree of enjoyment, sufficiency of information, promotion of memory, experience of sickness, degree of satisfaction, and propensity to purchase. These variables are used not only to evaluate the interfaces but also to distinguish between a "VO" and a "VE."

\section{A. Sense of Presence}

Sense of presence indicated how ready a user believed they could influence or interact with an object or an environment in the VE by moving and rotating the object therein. In other words, the user felt that they were in the perceived VE, or the perceived object was in their real surrounding. Based on Zeltzer's cube [12], attributes 
that differentiated a VE from a VR included levels of autonomy, interaction, and presence, among which sense of presence by far was the most important. Such a sense of presence measured the sense of "being in" an environment by calculating the number, fidelity, and appropriateness of the sensory input and output channels [12]. Therefore, to increase the sense of presence, researchers developed a variety of enhancing input and output devices to enrich the physical senses of a person, including head and body tracking, speech input, high-performance graphics (via photo-realism or virtual models), 3-D audio, and tactile/force feedback.

VR could be described as a mental experience where a user believed that "they are there" and "their mental experience" existed in the virtual world. By interacting with the environment, the user was no longer a mere observer of what was happening on the screen, but rather the user "felt" immersed in that world, in which they participated, despite the fact that such a world was only composed of spaces and objects existing in the memory of the computer and the user [10]. Further, the user no longer relied on a physical system, but their own psychological feelings to attain the sense of presence. Reference [21] showed that text-based VE offered a poor sense of presence; nevertheless, 69\% of 207 Multi-user Dungeon Dimension users felt the sense of presence.

Besides measuring sense of presence in textual medium, most researchers focused on comparison among devices in VE systems, whereas only few made comparison among media. Pausch et al. [22] quantified the immersion of a head-mounted display (HMD) and a stationary monitor, and showed that users with an immersive VR interface conduct searches faster than did those with a stationary monitor and a hand-based input device. Evidence also showed that HMD offered a better sense of presence compared with a desktop VR [6]. Johnson [5] conducted a survey on user responses to application of QTVR to museum Web pages, which were perceived by users as of better "feel for the object" and marginal improvement over text and 2-D graphics.

Since people who are familiar with the real 3-D world might find it easier to immerse in metaphoric representations (i.e., a higherdimensional level such as a VE) than in lower-dimensional representations (i.e., text and 2-D images), we have the following hypothesis.

Hypothesis 1 (H1): A 3-D VO has a higher rating in sense of presence than does a single 2-D image, a collection of 2-D images, or a photorealistic VR object.

\section{B. Level of Attraction}

Level of attraction referred to the degree to which users felt attracted to a presentation style. Attraction was a promotional strategy that drew users to Web sites; 3-D VOs could be utilized to promote such attraction, while Nielsen [1] warned against gratuitous use of 3-D VOs for attraction. Further, research [23] showed that the rich medium of VR storefront could engage customers for a longer period than could other online and kiosk formats. As a result, sales increased after the introduction of 3-D VOs that allow customers to do everything with the products except for smelling and touching [15]. Besides, Johnson [5] found that in a session where users had browsed through both conventional Web pages (text and 2-D images) and 3-D VRML models, users agreed that the latter was more attractive. Therefore, in terms of subjective experience, we have the following hypothesis.

Hypothesis 2 (H2): A 3-D VO has a higher rating in level of attraction than does a single 2-D image, a collection of 2-D images, or a photorealistic VR object.

\section{Degree of Enjoyment}

Degree of enjoyment reflected the level of pleasure that was brought by a presentation style to a user during their engagement with a product. Exciting, dynamic, and interactive display features, other than text descriptions and numeral specifications of a product on the Web, were capable of reducing monotonous feelings (or inducing enjoyment). By utilizing these displays, many Web sites attempted to hold the attention of customers long enough for the sales pitch [24]. Brown et al. [4] mentioned that one supporting staff enjoyed using VE so much that he still talked about the VE a month after using it. Johnson [5], with the perceived media held constant, proved that a casual browsing group experienced more enjoyment than a task-directed group, which indicates that enjoyment depended on the effect of task motivation. However, task motivation might not be the only independent variable that contributes to enjoyment. We have the following hypothesis.

Hypothesis 3 (H3): A 3-D VO has a higher rating in degree of enjoyment than does a single 2-D image, a collection of 2-D images, or a photorealistic VR object.

\section{Sufficiency of Information}

Sufficiency of information referred to the clarity and effectiveness of a perceived presentation style in providing users with fragmental details or as-a-whole information of a product. A picture might worth more than a thousand of words, but how many words was a 3-D VO worth? Wann and Mon-Williams [8] stated that "a VE provides the user with access to information that would not otherwise be available at that place or time." Visual information was not only a quantitative measure of spatial dimensions but also a natural extraction of environmental dimensions such as ordinal cues and relative body-scale information. Foreman et al. [25] found that the disabled children in their studies showed improvement of spatial skills only in a 3-D VE but not in a 2-D environment. Brown et al. [4] replicated the study by proving that an informative VE empowers people by allowing them to learn particular life skills at their own pace, that is, to learn in a representative context by reinforcing efforts and making mistakes.

Apart from comparison between 2-D displays and VE, other comparisons such as one between 2-D displays and 3-D graphics were carried out. Sutcliffe and Patel [7] stated that: "Graphics, and 3-D in particular, may not help more verbally inclined people, whereas the visualizers will benefit." The superiority of 3-D over 2-D display might vary [26], for instance, 3-D graphs were more efficient than 2-D in illustrating details, but 2-D graphs were more capable than 3-D in showing trends [27]. On one hand, the distortion inherent in 3-D displays hampered judgment in relative positions; on the other hand, integration of dimensions in 3-D displays facilitated shape understanding [28]. We have the following hypothesis.

Hypothesis 4 (H4): A 3-D VO has a higher rating in sufficiency of information than does a single 2-D image, a collection of 2-D images, or a photorealistic VR object.

\section{E. Promotion of Memory}

Promotion of memory referred to the ability of a presentation style to create short-term and long-term memories. One way to enhance marketing ability to impress users was to build an unforgettable scene on a 3-D display. However, full fidelity of VE was very costly, and its ability to promote memory remained uncertain. A comprehensive cognitive analysis of tasks should be performed in an attempt to serve as a useful basis for understanding the features of VE that might be preserved under different circumstances [29]. In fact, most of the cognitive analyses converged to VR projects on flat screens (desktop VR) or 3-D graphs.

According to Wickens' [30] argument on the basic of empirical data from a variety of sources, projects of VR on flat screens that 
enhance user's performance by reducing effort might actually inhabit long-term memory retention. Merwin and Wickens [31] found that a 2-D graphical interface led to worse performance but just as good retention as did the 3-D interface. Similar results were reported [32] for comparing a mono (less real) with a stereo (realism) view of scientific data. Levy et al. [27] found that a 3-D graph was easier to be memorized than a 2-D graph, but a 2-D graph was better to generate immediate impressions. Replicated results in [7] indicated that 3-D graphics helped to reduce representational complexity and promote memory, which showed 3-D graphical visualizations had an advantage over text in promoting gist memory of high-order information structures, and 3-D seemed to have a performance advantage for information retrieval.

Howes et al. [16] reported three experiments where participants were asked to search for products in various experimental electronic commerce (EC) sites in QTVR, hypertext, or pictorially rich hypertext environments. The result showed that participants who used the QTVR interfaces would have better memory for recalling products, which was incidental to their goals. Apart from quantitative measurements, we also believed that 3-D VOs may incite a better sense of first impression to users, and hence, we have the following hypothesis.

Hypothesis 5 (H5): A 3-D VO has a higher rating in promotion of memory than does a single 2-D image, a collection of 2-D images, or a photorealistic VR object.

\section{F. Experience of Sickness}

Experience of sickness referred to the users' physical and mental performances (whether they were suffering from sickness induced from VE such as nausea or otherwise) due to viewing and/or manipulating a presentation style. There were several health and safety issues in VE [33], [34]. Stanney et al. [34] identified both direct and indirect safety issues. Direct effects included physical trauma and sickness, whereas indirect effects included physiological aftereffects and disturbances. One of the most important direct effects that might affect the advancement of VE technology was cybersickness, where the user might endure various levels of sickness ranging from a slight headache to an emetic response.

While cybersickness was a commonly cited concern of humanvirtual environment interaction (HVEI), a lesser known yet equally important indirect consequence of HVEI was the potential deleterious physiological aftereffects from VE exposure [34]. Exposure to VE might produce disturbing aftereffects, such as head spinning, postural ataxia, reduced eye-hand coordination, and/or vestibular disturbances. It was therefore crucial to identify the relative sickness index, cybersickness, and aftereffects of VOs among various presentation styles, especially for individuals who may have protracted or repeated exposures to VOs during online shopping. Although subjective reports of motion sickness after VE exposure might provide little assistance in measuring an individual's perceived or experienced reduction in their physiological functioning [35], such reports still played an important role in measuring the direct effects. We believed that a VO (a close relative of a VE) might induce sickness in the perceivers, and therefore, we have the following hypothesis

Hypothesis 6 (H6): A 3-D VO has a higher rating in experience of sickness than does a single 2-D image, a collection of 2-D images, or a photorealistic VR object.

\section{G. Degree of Satisfaction}

The degree of satisfaction indicated the level of users' subjective state of pleasure with all aspects of a presentation style. One key objective of establishing Web-based e-catalogs was to provide better customer services and thereby to induce higher user satisfaction. Although not much research had been done on the nature of 3-D VOs of e-catalogs but rather on the applications of the same, where it was suggested that user satisfaction could be largely induced by interface quality or system performance.

From the perspective of interface quality, Howes et al. [16] demonstrated the effectiveness of photorealistic VR objects in leading users to a higher state of pleasure compared to hypertext and pictorial interfaces under certain conditions. Lewis and Pursglove [36] argued that the photorealistic VR technology resulted in an elevation of the user's subjective satisfaction as it provided an additional dimension than 2-D images. Further, Dysart [37] pointed out that "Customers have indicated that the availability of 3-D models can save up to two days of design work for each model they download." On the other hand, from the perspective of system performance, the users' subjective satisfaction depreciated, although to an insignificant extent, due to bandwidth consummation in retrieving 3-D VOs from the server [23]. As a result, we presumed that the individuals induced more satisfaction from 3-D VOs, and we have the following hypothesis.

Hypothesis 7 (H7): A 3-D VO has a higher rating in degree of satisfaction than does a single 2-D image, a collection of 2-D images, or a photorealistic VR object.

\section{H. Propensity to Purchase}

The propensity to purchase is related to the users' perception that they would purchase the observed products due to the presentation styles of a Web site in general and the object in specific provided that they had determined to purchase a product of a particular class. The objective of digitizing a business was to generate sales through the channel of the Internet, and thus, it was important to determine whether the presentation style of a product had any positive impact on the purchasing tendency of users. In fact, according to [23], there was no statistically significant difference in the money spent and items purchased between pictogram-based and VR storefronts. Moreover, Wilson [38] (in parallel with Wann and Mon-Williams [8]) doubted the helpfulness of the term "virtual reality" even when it was applied correctly, but more often, the term was used inappropriately for publicity and marketing purposes. Researchers in general did not agree that VR, which was a kind of 3-D display, could provoke purchase inclination.

From the practitioners' point of view, however, 3-D displays elevated the users' propensity to purchase. For example, eBay intended to offer users a variety of photorealistic VR-rich media services to enhance their listings, which results from the findings of iPIX Virtual Tours [53] that this media provided an important solution for the market by saving time and money as well as increasing transaction velocity for buyers and sellers [36]. Moreover, VE and VR were used for both offline and online sales and marketing. Over the past few years, Tyne and Wear Development Corporation and Real Time Design had adopted a VR system, i.e., Superscape, to construct and visualize the Newcastle Quayside development [39]. Sharper Image also made profit with the introduction of interactive 3-D VOs to its Web site [15]. Although this was a controversial issue, we have the following hypothesis.

Hypothesis 8 (H8): A 3-D VO has a higher rating in propensity to purchase than does a single 2-D image, a collection of 2-D images, or a photorealistic VR object.

\section{Participants}

Sixty participants (students and staff) were recruited for this study at the Hong Kong University of Science and Technology. Subjects, each having at least two years of experience in surfing the Web, included a 


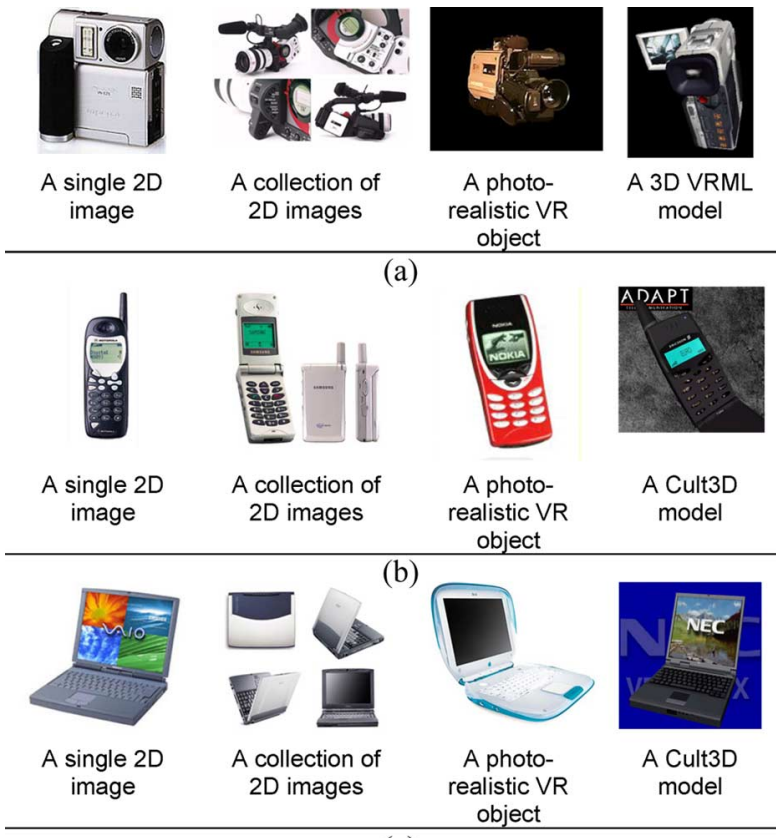

(c)

Fig. 1. Electronic products of (a) camcorder, (b) mobile phone, and (c) computer notebook shown in a form of a 2-D image, a collection of 2-D images, a photorealistic VR object, and a 3-D VO.

total of 40 males and 20 females with an overall average of 23.2 years in age. Twenty participants ( 12 males and 8 females) were assigned to the pilot test. As a token of appreciation, each subject was given 50 Hong Kong dollars (about US $\$ 6.5$ ) for their participation.

\section{J. Materials}

The materials used for this correspondence included an automated Web-based questionnaire and three products (camcorder, mobile phone, and notebook computer) introduced in four presentation styles (single 2-D image, collection of 2-D images, photorealistic VR object, and 3-D VO), as shown in Fig. 1. The 2-D images were in Joint Photographers Expert Group format. The photorealistic VR object was supported by software such as QTVR, which provided limited functions for users to rotate the picture of a photorealistic object $360^{\circ}$ vertically or horizontally. In a $3-\mathrm{D}$ VO, there were unlimited points of view where users were allowed to manipulate the model in whatever way they chose. In particular, users could activate and deactivate certain movement patterns (e.g., open and close the liquidcrystal display of the camcorder by pressing the button on the side of the panel) that were preset in the model.

The automated Web-based questionnaire included questions on age, gender, purchase experience on the Web, and participants' feedback after interacting with one of the products in four presentation styles. The questionnaire was divided into eight sections, each covering one response variable, and there were nine questions in each section for a total of 72 questions in the whole questionnaire. However, after the purification process based on the reliability coefficient analysis of the data collected from the pilot study, the total number of questions in the final questionnaire was reduced to 57 comprising of six to nine questions in each section (see the Appendix). In the pilot study, the resultant Cronbach's alpha values ranged from 0.84 to 0.95 with a modal point of 0.91 . In the entire study, the values ranged from 0.83 to 0.95 with a modal point of 0.91 . It was apparent that the levels of reliability were satisfactory, exceeding the commonly accepted level for exploratory research [40].

\section{K. Task and Procedure}

Each participant was required to view and manipulate a product in the four presentation styles and to fill in a questionnaire on the Web within a 1-h session. The automated Web system chose a presentation style of a particular electronic product and presented it to the participant in a scheduled counterbalanced order. Each participant was required to spend a maximum of $2-3$ min observing and manipulating each style before responding to the questionnaire. They were told to mark each response on a seven-point Likert scale with anchors from "strongly disagree (1)" to "strongly agree (7)." Data were then stored in a database via the Internet for further analysis. After rating a presentation style, another style was chosen by the automated system until all four styles were viewed, manipulated, and rated by each participant.

\section{Experimental Design}

A repeated one-way analysis-of-variance (ANOVA) procedure was used to predict whether the four different presentation styles would have any dissimilar responses in each of the variable, because the response variables were tested under four presentation styles by the same subjects. For example, in testing a response for "sense of presence" under four styles of presentation using ANOVA, the hypotheses could be expressed as $\mathrm{H}_{0}: \tau_{1}=\tau_{2}=\tau_{3}=\tau_{4}=0$ and $\mathrm{H}_{\mathrm{a}}$ : at least one $\tau_{i}$, for $i=1, \ldots, 4$, be different from zero, where $\mathrm{H}_{0}$ is the null hypothesis saying that the ratings of sense of presence among the presentation styles are the same to one another, and $\mathrm{H}_{\mathrm{a}}$ is an alternative hypothesis saying that the ratings of sense of presence of at least one presentation style among the four are dissimilar to each other. ANOVA is used as the analytical procedure to test the aforementioned hypotheses with the $F$-ratio, which can be manipulated based on the sum of squares of each effect and defined as

$$
F=\left(\sigma_{e}^{2}+\sigma_{\pi \tau}^{2}+n \sigma_{\tau}^{2}\right) /\left(\sigma_{e}^{2}+\sigma_{\pi \tau}^{2}\right)
$$

where $\tau$ is the effect of being in condition, $\pi \tau$ is the interaction, $e$ is the random error, and $n$ is the number of subjects. The degree of freedom, including the numerator and the denominator, is defined as ( $k-1, n-k$ ), where $k$ is the number of groups, and $n$ is the total number of observations. The $F$-test was used with $F$-ratio to decide to reject whether null hypothesis or alternative hypothesis.

In addition, Tukey's "Honestly Significantly Different" procedure was used for pairwise comparisons among different presentation styles, with comparison between 3-D VOs and the remaining styles being the exclusive focus. The procedure was based on comparing the critical value and the Tukey score $t$, which was defined as

$$
t=\left(M_{1}-M_{2}\right) / \sqrt{M S_{w}(1 / n)}
$$

where $M$ is the group mean, $M S_{w}$ is the Mean Square Within, and $n$ is the number per group. These procedures however were only applied if the mean ratings of each response were "near enough" to a normal distribution. The control factors included information presentation format and information content. The nuisance factor included the download time of individual Web pages, although that for each presentational style was nearly comparable.

In summary, the ANOVA procedure was used to predict whether the four different presentation styles would have any dissimilar responses in each variable, whereas Tukey's test was used for pairwise 
TABLE I

Descriptive Statistics (MeAn AND STANDARd DEviation) OF THE STUDY

\begin{tabular}{|c|c|c|c|c|c|}
\hline & & A & B & C & D \\
\hline \multirow{2}{*}{ Presence } & Mean & 3.19 & 3.62 & 4.70 & 5.11 \\
\hline & (SD) & $(0.95)$ & $(0.69)$ & $(0.78)$ & $(0.96)$ \\
\hline \multirow{2}{*}{ Attraction } & & 3.10 & 3.47 & 4.86 & 5.49 \\
\hline & & $(0.96)$ & $(0.78)$ & $(0.88)$ & $(0.82)$ \\
\hline \multirow{2}{*}{ Enjoyment } & & 2.91 & 3.26 & 5.01 & 5.80 \\
\hline & & $(1.02)$ & $(0.99)$ & $(0.92)$ & $(0.89)$ \\
\hline \multirow{2}{*}{ Information } & & 2.98 & 3.54 & 4.09 & 4.88 \\
\hline & & $(0.74)$ & $(0.80)$ & $(0.72)$ & $(0.68)$ \\
\hline \multirow{2}{*}{ Memory } & & 3.16 & 3.39 & 4.53 & 5.17 \\
\hline & & $(1.23)$ & $(1.01)$ & $(0.79)$ & $(0.93)$ \\
\hline \multirow{2}{*}{ Sickness ${ }^{a}$} & & -3.11 & -3.18 & -3.35 & -3.51 \\
\hline & & $(0.97)$ & $(0.89)$ & $(0.95)$ & $(0.99)$ \\
\hline \multirow{2}{*}{ Satisfaction } & & 3.12 & 3.54 & 4.75 & 5.40 \\
\hline & & (1.15) & $(1.09)$ & $(1.07)$ & (1.10) \\
\hline \multirow{2}{*}{ Purchase } & & 2.82 & 3.11 & 3.77 & 4.21 \\
\hline & & (1.01) & $(0.92)$ & $(0.99)$ & (1.16) \\
\hline Performance ${ }^{b}$ & & 2.27 & 2.59 & 3.55 & 4.10 \\
\hline
\end{tabular}

Note: "A", "B", "C" and "D" refer to single $2 \mathrm{D}$ image, collection of $2 \mathrm{D}$

images, photo-realistic VR object and 3D virtual object, respectively.

${ }^{\mathrm{a}}$ Lower index reveals higher experience of sickness.

${ }^{\mathrm{b}}$ Performance $=$ average of all mean values of a presentation style.

comparisons between the ratings of 3-D VOs and those of the other three presentation styles.

\section{ANALysis AND RESUlts}

In this correspondence, we first carried out analyses on the reliability of pilot data and experimental data, the outcomes of which indicated a satisfactory level of reliability. A cross tabulation of the presentation styles and responses was then illustrated by ways of mean and standard deviation in Table I and a 2-D column chart for visualizing the variable performances in Fig. 2. The mean ratings of each variable per presentation style were checked for normality. The Kolmogorov-Smirnov normality test was adopted for the goodness of fit, which was based on the comparison between the value of maximum deviation $D$ and its critical value. $D$ was defined as

$$
D=\max _{1 \leq i \leq N}\left|F\left(Y_{i}\right)-i / N\right|
$$

where $N$ is the number of observations, and $F$ is the cumulative frequency distribution of the random sample. The test result showed that except for response ratings in "attraction" for photorealistic VR objects and those in "enjoyment" for 3-D VOs, all remaining ratings

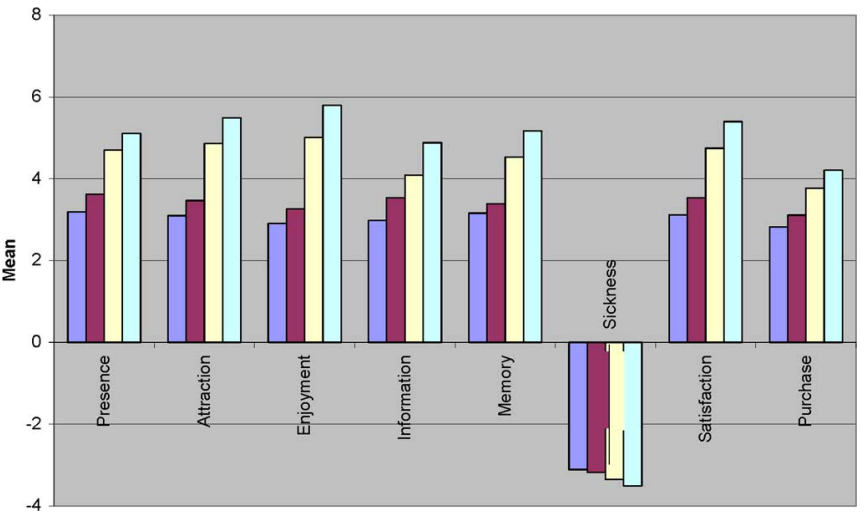

Fig. 2. Two-dimensional column chart of means of all response variables in four presentation styles. In each variable group, column bars are printed in a sequence of a single 2-D image, a collection of 2-D images, a photorealistic VR object, and a 3-D VO.

were normally distributed with $p$-values of no less than 0.01 , whereas a $p$-value greater than 0.15 was recommended. However, because the parametric tests such as ANOVA and Tukey's tests were fairly "robust," even when the assumptions of normal distribution could not be maintained, the parametric tests could still be valid unless the distribution of the mean ratings was very different from a normal distribution [41].

The main objective of this correspondence was to evaluate the performance of 3-D VOs in comparison with three other presentation styles with respect to the response variables, i.e., "presence," "attraction," "enjoyment," "information," "memory," "satisfaction," and "purchase." We found that the presentation style of 3-D VOs was superior in most cases. Starting with "presence," the null hypothesis was rejected; there were significant differences among the four presentation styles, $F(3,177)=73.31, p<0.0001$. The analysis continued with pairwise comparisons where a 3 -D VO significantly outperformed a single 2-D image $(t(6,234)=18.29, p<0.01)$, a collection of 2-D images $(t(6,234)=14.19, p<0.01)$, or a photorealistic VR object $(t(6,234)=3.92, p<0.05)$. As hypothesized, the participants perceived a higher level of "presence" from a 3-D VO compared with other presentation styles.

As for "attraction" $(F(3,177)=95.04, p<0.0001)$ and "enjoyment" $(F(3,177)=138.11, p<0.0001)$, it was revealed that significant differences existed among different presentation styles. A post hoc Tukey's test showed that 3-D VOs offered higher and more significant attraction to participants than did a single 2-D image $(t(6,234)=$ $20.58, p<0.01)$, a collection of 2-D images $(t(6,234)=17.41$, $p<0.01)$, or a photorealistic VR object $(t(6,234)=5.40, p<0.01)$. Similarly, from the post hoc test, the participants experienced greater enjoyment with the interactive features of a 3-D VO than with a single 2-D image $(t(6,234)=24.51, p<0.01)$, a collection of 2-D images $(t(6,234)=21.57, p<0.01)$, or a photorealistic VR object $(t(6,234)=6.71, p<0.01)$.

In terms of cognitive-related responses, different presentation styles performed differently in delivering information to perceivers $(F(3,177)=114.11, p<0.0001)$ and in promoting incidental memory $(F(3,177)=60.12, p<0.0001)$. Participants indicated that they perceived clearer and more complete information as well as learned additional knowledge through a 3-D VO than through a single 2-D image $(t(6,234)=25.04, \quad p<0.01)$, a collection of 2-D images $(t(6,234)=17.65, p<0.01)$, or a photorealistic VR object $(t(6,234)=10.35, p<0.01)$. Moreover, the participants indicated that a 3-D VO could stimulate user's incidental memory more efficiently and effectively, and the portrayed image of a 3-D VO was 
retained in the users' brain for much longer than that of a single 2-D image $(t(6,234)=16.37, p<0.01)$, a collection of 2-D images $(t(6,234)=14.53, p<0.01)$, or a photorealistic VR object $(t(6,234)=5.18, p<0.01)$.

Regarding "sickness," evidence was obtained that there was at least one presentation style that significantly differed from the others, which caused the null hypothesis to be rejected $(F(3,177)=6.97$, $p=0.0002)$. The participants reported little "sickness" or even no "sickness" (since the neutral value is 4) from all the image-based and object-based presentation styles. The differences in mean between a single 2-D image and a collection of 2-D images, between a collection of 2-D images and a photorealistic VR object, and between a photorealistic VR object and a 3-D VO were no more than 0.2 with rated total mean of 3.29 and standard deviation of 0.96 . Yet, the participants assessed a 3-D VO to be a style inducing "sickness" at a level that was relatively higher than that of a single 2-D image $(t(6,234)=$ $5.91, p<0.01)$ and a collection of 2-D images $(t(6,234)=4.80$, $p<0.01)$. A simple contrast between a 3-D VO with a photorealistic VR object gave no significant difference $(t(6,234)=2.31, p>0.05)$.

Finally, "satisfaction" and "purchase" were the psychological responses related to users' inclination to purchase. Repeated one-way ANOVA revealed significant differences in "satisfaction" and "purchase" among different style conditions with $F(3,177)=69.7, p<$ 0.0001 and $F(3,177)=49.48, p<0.0001$, respectively. Tukey's comparison found that a $3-\mathrm{D}$ VO provided a significantly higher degree of "satisfaction" than did a single 2-D image $(t(6,234)=18.03$, $p<0.01)$, a collection of 2-D images $(t(6,234)=14.71, p<0.01)$, or a photorealistic VR object $(t(6,234)=5.15, p<0.01)$. As for the responses of "purchase," participants also found that they were more inclined to purchase the perceived items based on a 3-D VO rather than a single 2-D image $(t(6,234)=15.51, p<0.01)$, a collection of 2-D images $(t(6,234)=12.28, p<0.01)$, or a photorealistic VR object $(t(6,234)=4.89, p<0.01)$.

\section{DISCUSSION}

The experimental results demonstrated that, in a laboratory context, e-catalogs using 3-D VOs offered significant advantages over those based on hypertext with a single 2-D image, a collection of 2-D images, or a photorealistic VR object. There was evidence that participants maneuvering in a 3-D VO environment had a better sense of "feel for the object," experienced a higher degree of attraction and enjoyment, perceived more information, retained much longer inherited reflection through incidental observation, achieved more satisfaction, became more inclined to purchase, and were more apt to feel ill. These findings were crucial from both theoretical and practical perspectives.

\section{A. Theoretical Perspective}

From a theoretical perspective, the results added sound findings to the study of hierarchical relationships within the domain of presentation styles, particularly on differences between VOs and VEs, in the existing conceptual frameworks. That is, to understand how the response variables of a presentation style in terms of the user's subjective ratings related to the components of VR addressed by Wickens and Baker [29], as well as the Zeltzer's cube [12], although these tools were assumed to apply only to VR and its related systems. The aim of this discussion was to enable a quick reasonable judgment on the subjective performances of a presentation style with representative indices.

Wickens and Baker [29] considered that VR was not a unified thing but could be broken down into a set of five features, any one of which could be present or absent in creating a greater sense of reality. The components of VR, i.e., a five-element tuple in nominal measurement
TABLE II

COMPONENTS OF ViRTUAL REALity (From Wickens AND BAKER [29])

\begin{tabular}{lcc}
\hline & "0" & "1" \\
\hline Dimensionality & $2 \mathrm{D}$ & $3 \mathrm{D}$ \\
Motion & Static & Dynamic \\
Interaction & Open loop & Closed loop
\end{tabular}

Frame of reference World-referenced Ego-referenced

Multi-modal interaction Limited Multi-modal

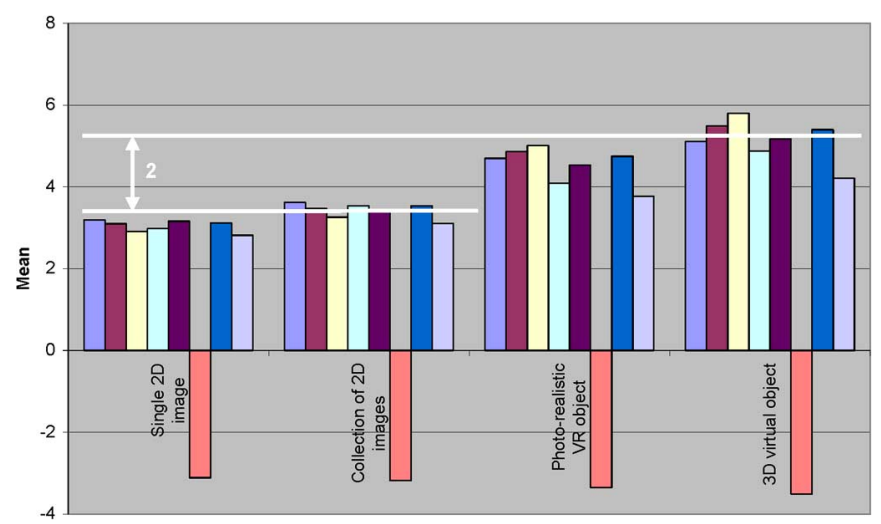

Fig. 3. Two-dimensional column chart arranged by presentation style. In each presentation style group, column bars are printed in a sequence of "presence," "attraction," "enjoyment," "information," "memory," "sickness," "satisfaction," and "purchase."

of 0 and 1, were dimensionality, motion, interaction, frame of reference, and multimodal interaction (see Table II). By applying this framework to the presentation style of a 3-D VO instead of the usual VR or VE systems, a single or a collection of 2-D image(s) can be described as a tuple of $(0,0,0,0,0)$ in which the five 0 s refer to 2 -D view, static display, open loop interaction, world referenced (or outside-in frame-of-reference), and limited multimodal interaction, respectively.

Similarly, a photorealistic VR object and a 3-D VO could be denoted as a tuple of $(1,1,1,0,0)$, where these two presentation styles were at least in 3-D view dynamic movement with closed-loop interaction but still in a world-referenced contrast and suffering from limited multimodal interaction of keyboards and mice. The four presentation styles could consequently be divided into two groups of tuples $(0,0$, $0,0,0)$ and $(1,1,1,0,0)$. While observing the descriptive statistics plotted in Fig. 3, the presentation styles could also be classified into an image group and an object group. In terms of aggregated "performance" in Table I, in which the numerical index was comparatively representative, there was a large difference between the image group and the object group, while only small differences were found within each group. These findings confirmed that the components of VR in [29] provided a basic indication of performances of a presentation style.

Zeltzer's cube [12] with its three ordinal measurement axes of autonomy, interaction, and presence provided a conceptual tool for organizing our understanding of the current VE technology. Applying this scheme to the presentation style of a single or a collection of 2-D image(s), the style can be described as $(0,0,0)$ and classified as 


\begin{tabular}{|c|c|c|c|c|c|c|c|c|c|c|}
\hline \multicolumn{6}{|c|}{ Wickens and Baker [29] } & \multirow[t]{2}{*}{ Presentation mode } & \multicolumn{4}{|c|}{ Zeltzer's [12] } \\
\hline $\mathrm{D}$ & M & 1 & $\mathrm{~F}$ & MI & $\mathrm{Ag}^{\mathrm{a}}$ & & $\mathrm{Ag}^{\mathrm{b}}$ & A & I & $P$ \\
\hline$\square$ & $\square$ & $\square$ & $\square$ & $\square$ & 0 & A single $2 \mathrm{D}$ image & 0 & $\square$ & $\square$ & $\square$ \\
\hline$\square$ & $\square$ & $\square$ & $\square$ & $\square$ & 0 & A collection of $2 \mathrm{D}$ images & 0 & $\square$ & $\square$ & $\square$ \\
\hline - & - & घ & $\square$ & $\square$ & 3 & A photo-realistic VR object & $1 / 2$ & $\square$ & - & $\square$ \\
\hline - & घ & - & $\square$ & $\square$ & 3 & A 3D virtual object & $11 / 2$ & - & $\mathbf{\square}$ & $\square$ \\
\hline - & - & घ & च & च & 5 & A virtual environment & 2 & $\square$ & घ & घ \\
\hline - & - & घ & $\square$ & - & 5 & A virtual reality & 3 & - & घ & घ \\
\hline
\end{tabular}

Note: $\square=0, \cdot=1 / 2$, and $\mathbf{\square}=1$.

${ }^{a}$ Aggregated performance of dimensionality (D), motion (M), interaction (I), frame of reference $(\mathrm{F})$, and multi-modal interaction (MI).

b Aggregated performance of autonomy (A), interaction (I), and presence $(P)$.

Fig. 4. Analogical hierarchical pyramid of presentation modes based on Wickens and Baker's indices [29] and Zeltzer's cube [12].

a model with no autonomy, no interaction, and no presence. Since the cube is made up of vectors differing from the binary components of VR in [29], we define " 0 ," " $1 / 2$," and " 1 " as no, partial, and full capacity, respectively. Thus, a photorealistic VR object could be represented by $(0,1 / 2,0)$ due to its finite degree of interaction as it restricted users to spin the object only. On the other hand, a 3-D object provided full interaction functions (e.g., tilt, slide, move, and rotate with full degrees) to users. However, a VO had a restricted ability to act and react to simulate and therefore be decoded as $(1 / 2,1,0)$.

Combining the above arguments with the research findings, we came to an interesting result. If the models above were arranged into hierarchical orders on the basis of consolidated vectors, the image group with zero value would be positioned at the bottom, while a 3-D VO (equivalent to 11/2) would be on the top, and a photorealistic VR object (equivalent to $1 / 2$ ) in the middle. A similar hierarchical pattern was also found in the statistical results that the a 3-D VO had advantageous capacity over a single 2-D image, a collection of 2-D images, and a photorealistic VR object under most of the settings. Moreover, when comparing individual responses or aggregated "performance" in Table I with one another, 3-D VOs were the best, followed by the photorealistic VR objects, and then plain images (see Fig. 4). The overall findings, from our statistical results to the conceptual tools indices of [12] and [29], had shaped these presentation styles into a certain well-defined hierarchical structure and shown that the judgment of the presentation style performances was possible in association with these conceptual tools.

Furthermore, we quantified performances of VE and VR with the tools of [12] and [29]. VE performed similarly with VR that could be explicated as a tuple of $(1,1,1,1,1)$ using the components of VR [29]. That is, in addition to a 3-D view and a dynamic movement with closed-loop interactions, the system had the ability to project from the perspective of the point of ego reference of the users and to employ a variety of techniques for the users' input. However, from Zeltzer's [12] point of view, VE and VR were unlike and were defined as $(0,1,1)$ and $(1,1,1)$, respectively. According to the analogy of conceptual tool indices to measure presentation style performances, we concluded that the "virtual" group should have better performances than the object group and image group, and the VR should be on the top of the VE.

In order to test if our analogical hierarchical structure was on the right course, particularly the sequence of VR, VE, and 3-D VOs, we tried to validate this structure with the existing literature. Unfortunately, there was little research on the comparison of VR and VE [12], and of VE and 3-D VOs [6], [22]. The lack of research on
VR was understandable since it was located at an unattainable node within the current technology [12]. There was not a model that had full autonomy nor a system with full interaction and full presence, and hence, research on this issue should be very limited. No better than the prior facts, the comparison of VE and 3-D VOs was bounded by the topic of immersion. This was also reasonable since the vector of presence between 3-D VOs and VE changed from 0 to 1 , which is a major change from no to full presence. The change of magnitude from 0 to 1 in presence indeed had been verified by research [6], [22] that an HMD increased the sense of presence in the same direction with the magnitude over a desktop VR, which further proved our analogical hierarchical pyramid.

\section{B. Practical Perspective}

At first glance, it was hard to tell that the use of subjective ratings in our experiment, particularly that of the response variables of "sufficiency of information" and "promotion of memory," was comparable to task-based experiments; but indeed, the findings replicated results with research using objective measurements. For example, St. John et al. [28] measured reaction time, total search time, and error from the participants objectively for the analysis in "sufficiency of information." Smallman et al. [26] did a computer application to record the screen locations and times of all mouse clicks. In terms of "promotion of memory," Howes et al. [16] measured objective responses using the number of items successfully located, average nodes visited per task, average time taken per task(s), etc. These findings in objective measurements, to some extent, were similar to our findings with subjective ratings, although the experiments were carried out in different subject areas. We believed that the subjective ratings were representative and as useful as objective measurements.

But what if our subjective ratings were incomparable to the objective measurements of other people's work? Would these skewed findings still be representative and usable? Based on our findings, the users had a relative bias toward purchasing products that were displayed in 3-D VO. Given this condition, the actual performance of a medium in e-catalogs was not critical at all, although it might be important to other areas such as education and training. Nevertheless, the subjective feelings of majority buyers who purchased through a particular presentation style were the main concerns of real business world. In addition to "propensity to purchase," other variables were also applicable to business functions especially in sales and marketing.

Turning the attention to implementation, we found that most Web sites made limited use of 3-D interactive perception views in e-catalogs despite our results indicating that it was worthwhile to employ a highlevel structural perspective in exhibiting extrinsic-oriented products. For example, referring to Fig. 2, in general, the examined presentation styles on account of individual responses (except "experience of sickness") were mainly divided into groups of "image" and "object" (see Fig. 3). The object group of photorealistic VR objects and 3-D VOs represented a sharp improvement in performances over the image group of a single or a collection of 2-D image(s). The mean difference between the performances of these two groups was approximately two units. As a result, while the Web consumers would perceive that it was more effective to use the object group in many senses, the implementers would doubt the cost effectiveness of doing so. In fact, parallel with Howes et al. [16], we agreed that the amount of investment in constructing a photorealistic VR object Web site was much higher than that in creating a hypertext environment.

Opposite results, however, from recent research [42] suggested that using 3-D VOs specifically for portraying customized products had enormous advantages in development time, required cost, and storage space over existing catalogs (static-, paper-, and image-based catalogs) 
under most conditions. The assessment was based on properties of dynamic, online, and 3-D presentation modes, and was carried out from the standpoint of companies and users. In summary, a cataloging system of a 6000 -combination 3-D VO saved $23 \%$ of the total development time of an image-based catalog and $99 \%$ of the total storage space a hard drive, but more or less the same amount of the total required cost. Online retailers needed sound reasons before they could make their decision to invest, but initial evidence showed that implementation of 3-D VOs was quite cost effective for customized products.

\section{CONCLUSION}

While immersive systems tended to be favored in entertainment and promotional applications, nonimmersive systems (desktop VR) were preferred in applications requiring higher fidelity and continuous use [39]. As Lowe [39] suggested, desktop VRs were often utilized on the Web in relation to sales and marketing [14], [18], [23], education [4], [30], data visualization [7], [26]-[28], and so on. It was one of the most interesting developments for Web-based EC to deploy catalogs on the Web, where content of e-catalogs migrated from 2-D images to 3-D VOs, which was not only a viewing technique to facilitate consumers to see the products online but also an intelligent tool that allowed users to customize their products on the display. Further, the benefits of VRML went beyond making fascinating games [43]. However, before 3-D VOs could become fully utilized for e-catalogs, an appropriate tool for assessing the usability of such 3-D VO system must be established.

The direction of existing and future research in human-computer interaction should no longer be confined to interface or display but extended to sense of touch [44]-[46] and smell [47]. For instance, with the invention of PHANTOM [46], touching a VO was no longer impossible. Users could touch the VOs through the "haptic" interface that appeared as something physical. DigiScents iSmell [54] was a digital scent technology that brought the sense of smell to your computer. Users could immerse themselves in scented movies or entertainment on the Web or sniffed before buying a product online without leaving their seats. And if these technologies could be integrated to form a system for experiencing a product through different senses, then it would certainly provide users with more sophisticated "tangible" information on products together with easy access features, which represented a tremendous breakthrough in e-catalogs as a tool of sales and marketing.

To conclude, the results of this correspondence indicated that 3-D VOs were superior to images and photorealistic VR objects in many aspects. In addition to the advantages of human-computer interactions of a 3-D VO cataloging system performance, the textual properties of VRML had led 3-D VOs to a more cost-effective position, i.e., a much smaller investment to portray 3 -D customized products than to create pictorially rich hypertext environments [42]. Further, these findings derived a presentation style performance quantifying tool in [12] and [29]. However, 3-D VOs had many limitations within the existing technology, for example, users' experience of a higher level of sickness, occupation of the bandwidth for complex 3-D models, and lack of supporting software.

\section{APPENDIX}

\section{INSTRUMENT USED IN THIS CORRESPONDENCE}

\section{A. Sense of Presence (Pilot $\alpha=0.84$; Experimental $\alpha=0.85$ )}

1) I got a sense of presence of the object is "here."

2) This way of presentation reduced my feeling of presence.

3) I thought that the field of view enhanced my sense of presence.

4) The display quality reduced my sense of presence.
5) I felt isolated and not part of the VE.

6) The display appeared to be flat and lacking depth.

7) I would rate my sense of presence as "very satisfactory."

\section{B. Level of Attraction (Pilot $\alpha=0.92 ;$ Experimental $\alpha=0.91$ )}

1) This type of presentation increased the attractiveness.

2) I prefer to use this presentation style.

3) This one did not attract me very much.

4) The image/model shown locked my view.

5) I do not think the mode of manipulation attracted me very much.

6) I was impressed with the presentation style.

7) I am not willing to spend most of the time using this presentation style.

8) This style is the one that I would like to use to view/manipulate the object.

9) I would rate my level of attraction as "very satisfactory."

\section{Degree of Enjoyment (Pilot $\alpha=0.93$; Experimental $\alpha=0.95$ )}

1) I enjoy the interactive features very much.

2) If I had time, I would like to play this more.

3) It is very likely that I will choose this style next time.

4) This presentation style reduced my degree of enjoyment.

5) I enjoyed this style of presentation.

6) I am still excited about this presentation style after I finished viewing/manipulating the object.

7) I would rate my degree of enjoyment as "very satisfactory."

\section{Sufficiency of Information (Pilot $\alpha=0.90$; Experimental} $\alpha=0.90)$

1) This presentation style provides me with the most complete information.

2) I believe that I did learn something from this style.

3) I think I have learned how to use the product after the scrutiny.

4) It was difficult to perceive what functions were available.

5) I understood the product specifications through this style.

6) This style does not provide clear information.

7) I would rate my sufficiency of information as "very satisfactory."

\section{E. Promotion of Memory (Pilot $\alpha=0.93$; Experimental $\alpha=0.92$ )}

1) I have forgotten what it looks like right away.

2) I have easily memorized the whole structure of the product.

3) This presentation style increased my ability to memorize.

4) I believe that I would not remember the structure within a minute.

5) I got a clear picture of this style in my memory for a certain period.

6) This style was not effective in stimulating my memory.

7) I still remember the shape, style, and color of the product.

8) I would rate my promotion of memory as "very satisfactory."

\section{F. Experience of Sickness (Pilot $\alpha=0.85$; Experimental $\alpha=0.83$ )}

1) I felt uncomfortable after viewing and manipulation.

2) This style caused me some psychological discomfort.

3) When viewing/manipulating the interface, I did take a rest for a while.

4) I am tired of using this style.

5) I got a bit dizzy during viewing and manipulation.

6) I would rate my experience of sickness as "very high/sick." 


\section{G. Degree of Satisfaction (Pilot $\alpha=0.95$; Experimental $\alpha=0.93$ )}

1) I feel as if this style was well designed to meet my needs.

2) This style is the best way to present this product.

3) If I could play it over again, I would not use this style.

4) The way the style was introduced is my best choice.

5) I would like to introduce this style of presentation to my friend.

6) If I had to inspect a product, it is very likely that I would use this style.

7) I would rate my extent of satisfaction as "very satisfactory."

\section{H. Propensity to Purchase (Pilot $\alpha=0.88$; Experimental $\alpha=0.88$ )}

1) I would like to purchase this item due to the presentation style.

2) If I purchased an item right now, I would purchase this item.

3) If I had the money available, I would not purchase this item.

4) I would definitely like to purchase this item.

5) This style did not make me more inclined to purchase this item.

6) I would rate my propensity to purchase as "very satisfactory."

\section{ACKNOWLEDGMENT}

The authors would like to thank the editors and anonymous reviewers whose valuable suggestions have made substantial improvements to this correspondence.

\section{REFERENCES}

[1] J. Nielsen, Usability Engineering. Boston, MA: Academic, 1993.

[2] J. Nielsen, "Estimating the number of subjects needed for a thinking aloud test," Int. J. Human-Comput. Stud., vol. 41, no. 3, pp. 385-397, 1994.

[3] L. A. Miller and K. M. Stanney, "The effect of pictogram-based interface design on human-computer performance," Int. J. Hum.-Comput. Interact., vol. 9, no. 2, pp. 119-131, 1997.

[4] D. Brown, H. Neale, S. Cobb, and H. Reynolds, "Development and evaluation of the virtual city," Int. J. Virtual Real., vol. 4, no. 1, pp. 28-41, 1999.

[5] C. Johnson, "On the problems of validating desktopVR," in Proc. HCI-People and Computers XIII, H. Johnson, L. Nigay, and C. Roast, Eds., 1998, pp. 327-338.

[6] S. Nichols, C. Haldane, and J. R. Wilson, "Measurement of presence and its consequences in virtual environment," Int. J. Hum.-Comput. Stud., vol. 52, no. 3, pp. 471-491, 2000.

[7] A. Sutcliffe and U. Patel, "3-D or not 3D: Is it nobler in the mind," in Proc. HCI-People and Computers XI, M. A. Sasse, R. J. Cunningham, and R. L. Winder, Eds., 1996, pp. 79-94.

[8] J. Wann and M. Mon-Williams, "What does virtual reality NEED?: Human factors issues in the design of three-dimensional computer environment," Int. J. Hum.-Comput. Stud., vol. 44, no. 6, pp. 829-847, 1996.

[9] P. Zhang, R. V. Small, G. M. von Dran, and S. Barcellos, "Websites that satisfy users: A theoretical framework for Web user interface design and evaluation," in Proc. HICSS-32, 1999, p. 2016. 00012016.pdf.

[10] G. Riva, "From technology to communication: Psycho-social issues in developing virtual environments," J. Vis. Lang. Comput., vol. 10, no. 1, pp. 87-97, 1999.

[11] R. S. Kalawsky, The Science of Virtual Reality and Virtual Environments: A Technical, Scientific and Engineering Reference on Virtual Environments. Workingham, U.K.: Addison-Wesley, 1993.

[12] D. Zeltzer, "Autonomy, interaction, and presence," Presence: Teleoperators Virtual Environ., vol. 1, no. 1, pp. 127-132, 1992.

[13] S. V. G. Cobb, S. C. Nichols, A. R. Ramsey, and J. R. Wilson, "Virtual reality-induced symptoms and effects (VRISE)," Presence: Teleoperators Virtual Environ., vol. 8, no. 2, pp. 169-186, 1999.

[14] R. R. Burke, "Virtual shopping: Breakthrough in marketing research," Harvard Bus. Rev., vol. 74, no. 2, pp. 120-131, 1996.

[15] L. Goff, "The sharper image," Catalog Age, pp. S15-S16, Aug. 1999.

[16] A. Howes, G. E. Miles, S. J. Payne, C. D. Mitchell, and A. J. Davies, "Incidental memory and navigation in panoramic virtual reality for electronic commerce," Hum. Factors, vol. 43, no. 2, pp. 239-254, 2001

[17] G. E. Miles, A. Howes, and A. Davies, "A framework for understanding human factors in Web-based electronic commerce," Int. J. HumanComput. Stud., vol. 52, no. 1, pp. 131-163, 2000.
[18] B. P.-C. Yen and K. Y. M. Ng, "Web-based virtual reality catalog in electronic commerce," in Proc. HICSS-33, 2000, p. 6037. INMIW03.pdf.

[19] B. P.-C. Yen and K. Y. M. Ng, "Electronic catalog for personalized mobile phone and its impact," in Proc. ICEC, 2001, pp. VIII.2.1-VIII.2.6.

[20] J. Preece and B. Shneiderman, "Survival of the fittest: The evolution of multimedia user interfaces," ACM Comput. Surv., vol. 27, no. 4, pp. 557$559,1995$.

[21] J. Towell and E. Towell, "Presence in text-based networked virtual environments or 'MUDS'," Presence: Teleoperators Virtual Environ., vol. 6 , no. 5 , pp. 590-595, 1997.

[22] R. Pausch, D. Proffitt, and G. Williams, "Quantifying immersion in virtual reality," in Proc. 24th Annu. Conf. Comput. Graph. and Interactive Tech., 1997, pp. 13-18.

[23] J. C. Westland and G. Au, "A comparison of shopping experiences across three competing digital retailing interfaces," Int. J. Electron. Commer. vol. 2, no. 2, pp. 57-69, 1998.

[24] T. D. C. Little, "Commerce on the Internet," IEEE Multimedia, vol. 1, no. 4, pp. 74-78, Dec. 1994.

[25] N. Foreman, P. Wilson, and D. Stanton, "VR and spatial awareness in disabled children," Commun. ACM, vol. 40, no. 8, pp. 76-77, Aug. 1997.

[26] H. S. Smallman, M. St. John, H. M. Oonk, and M. B. Cowen, "Information availability in 2D and 3D displays," IEEE Comput. Graph. Appl., vol. 21, no. 5, pp. 51-57, Sep./Oct. 2001

[27] E. Levy, J. Zacks, B. Tversky, and D. Schiano, "Gratuitous graphics? Putting preferences in perspective," in Proc. CHI, 1996, pp. 42-49.

[28] M. St. John, M. B. Cowen, H. S. Smallman, and H. M. Oonk, "The use of $2 \mathrm{D}$ and $3 \mathrm{D}$ displays for shape-understanding versus relative-position tasks," Hum. Factors, vol. 43, no. 1, pp. 79-98, 2001.

[29] C. D. Wickens and P. Baker, "Cognitive issues in virtual reality," in Virtual Environments and Advanced Interface Design, W. Barfield and T. Furness, III, Eds. New York: Oxford Univ. Press, 1995, pp. 514-541.

[30] C. D. Wickens, "Virtual reality and education," in Proc. IEEE Int. Conf. Syst., Man and Cybern., New York, 1992, pp. 842-847.

[31] D. H. Merwin and C. D. Wickens, "2D vs. 3D display for multidimensional data visualization: The relationship between task integrality and display proximity," in Proc. 35th Annu. Meeting Human Factors Soc., San Francisco, CA, 1991, pp. 388-392.

[32] C. D. Wickens, D. H. Merwin, and E. Lin, "The human factors implications of graphics enhancements for the visualization of scientific data: Dimensional integrality, stereopsis, motion, and mesh," Hum. Factors, vol. 36, no. 1, pp. 44-61, 1994.

[33] E. Viire, "Health and safety issues for VR," Commun. ACM, vol. 40, no. 8 , pp. 40-41, Aug. 1997.

[34] K. M. Stanney, R. R. Mourant, and R. S. Kennedy, "Human factors issues in virtual environments: A review of the literature," Presence: Teleoperators Virtual Environ., vol. 7, no. 4, pp. 327-351, 2001.

[35] K. S. Kennedy and K. M. Stanney, "Postural instability induced by virtual reality exposure: Development of a certification protocol," Int. J. Hum. Comput. Interact., vol. 8, no. 1, pp. 25-47, 1996.

[36] E. Lewis and K. Pursglove, eBay Selects iPIX to Power Imaging on World's Leading Online Person-to-Person Trading Site, 2000, Reston, VA: Internet Pictures Corporation. [Online]. Available: http://www.ipix.com/aboutus/press/042000.html

[37] J. Dysart, "AMP adds 3-D parts to its Web catalog," Advertising Age's Business Marketing Online, 1997. [Online]. Available: http://www. netb2b.com/cgi-bin/netb2b/article.pl?id=637

[38] J. R. Wilson, "Virtual environments applications and applied ergonomics," Appl. Ergon., vol. 30, no. 1, pp. 3-9, 1999.

[39] R. Lowe, "Three UK case studies in virtual reality," Virtual Real. World, vol. 2, no. 2, pp. 51-54, 1994.

[40] J. C. Nunnally, Psychometric Theory, 2nd ed. New York: McGraw-Hill, 1978.

[41] J. Greene and M. d'Oliveria, Learning to Use Statistical Tests in Psychology, 2nd ed. Buckingham, U.K.: Open Univ. Press, 1999.

[42] B. P.-C. Yen and K. Y. M. Ng, "Development and evaluation of dynamic virtual object catalogs," Inf. Manage., vol. 40, no. 4, pp. 337-349, Mar. 2003.

[43] T. Gorman, VRML on Brink of Mainstream Acceptance Says VRML Cofounder Tony Parisi, 1997, Mountain View, CA: Netscape World. [Online]. Available: www.netscapeworld.com/netscapeworld/nw-071997/nw-07-vmrl.t.html

[44] D. A. Bowman and L. F. Hodges, "Formalizing the design, evaluation, and application of interaction techniques for immersive virtual environments," J. Vis. Lang. Comput., vol. 10, no. 1, pp. 37-53, 1999. 
[45] R. W. Lindeman, J. L. Sibert, and J. K. Hahn, "Toward usable VR: An empirical study of user interfaces for immersive virtual environments," in Proc. CHI, 1999, pp. 64-71.

[46] J. K. Salisbury, "Making graphics physically tangible," Commun. ACM, vol. 42, no. 8, pp. 74-81, Aug. 1999.

[47] K. Maney, Net is Getting Weirder, 2000, McLean, VA: USA Today. [Online]. Available: www. usatoday.com/life/cyber/ccarch/cckev004.htm

[48] Lands' End. [Online]. Available: www.landsend.com/
[49] Charles Tyrwhitt. [Online]. Available: www.tyrwhitt.com/

[50] The Sharper Image. [Online]. Available: www.sharperimage.com/

[51] Apple-Hardware-iBook. [Online]. Available: www.apple.com/ibook/ qtvr1.html

[52] Lunatic Interactive. [Online]. Available: www.lunatic.de/content/vrml/ sony/sony_cam.htm

[53] Internet Pictures Corporation. [Online]. Available: www.ipix.com/

[54] Digicents iSmell. [Online]. Available: www.digiscents.com/ 